\title{
Orlando nursing process based healthcare information management system
}

\author{
Adegboye Adegboyega ${ }^{1}$, Akpan Julius Aniefiok ${ }^{2}$ \\ ${ }^{1}$ Dept. of Maths \& Statistics, Computer \& Information System, Achievers University, Owo Nigeria \\ ${ }^{2}$ Federal Ministry of works, Abuja, Nigeria
}

Email address:

akanbi2090@yahoo.co.uk (A. Adegboyega), juliuswhiz@gmail.com (A. J. Aniefiok)

\section{To cite this article:}

Adegboye Adegboyega, Akpan Julius Aniefiok. Orlando Nursing Process Based Healthcare Information Management System. American Journal of Software Engineering and Applications. Vol. 3, No. 5, 2014, pp. 56-62. doi: 10.11648/j.ajsea.20140305.11

\begin{abstract}
The processes and management of healthcare records are not trivial exercise; there is need for application of ICT to healthcare management system in order to meet globally accepted health care systems. Many healthcare systems have been designed and implemented, but they do not adequately incorporate nursing process. Also, most of them do not consider the needs and aspirations of patients. In this work, a modified framework based on Orlando's nursing process focuses on improvement in the patient's behavior by actions that are based on a patient's needs found through effective interaction with the patient was designed and proto type implemented. The design was implemented using Visual Basic.Net and SQL because of their supports for implementing web-based systems. The system was hosted on a website for a period of two months. Real life data in respect of medical practitioners' and patients were captured, analyzed and evaluated. Users interacted with the hosted system during the evaluation period. The system was evaluated using usability test and structured questionnaire. The result showed $93.10 \%$ participation efficiency, while ease of use, operational efficiency and data protection of Healthcare Information system scored more than $80 \%$.This showed that the Healthcare Information Systems (HIS) is an effective life saving system that can influence and enhance health-workers' quality of services, timely precision decision making process and reduced cost of health care significantly through effective healthcare management. It is applicable in any healthcare environment irrespective of their social economic and technology settings. The application of the framework will prevents the spread of deadly diseases like Ebola Virus.
\end{abstract}

Keywords: Information and Communication Technology (ICT), Healthcare Information System Healthcare Records, Orlando's Nursing Process, Healthcare Management System and Ebola Virus

\section{Introduction}

The processes and management of healthcare records are not trivial exercise. Healthcare Information Systems will play an important role in providing patient information to physicians, nurses and administrative staff, and can be a significant factor in developing cooperation among physicians with regard to sharing healthcare information [Yang and Li, 2010, Yang et al., 2011]. Medical practitioners' needs accurate and timely information concerns patients in order to save life, and performed their duties professionally. The traditional manual method of recording, administer drug and billing patient can no longer meets today patient's medical attentions and lack transparency, since medical care is a real time information-intensive activity. The application of (ICT) to Hospital Management will enhance service delivery, streamlined operations, enhanced administration and control, superior patient care, strict cost control, improved profitability, data security and meet globally accepted health care systems. Today's Healthcare Information are in high demand in order to handle increasing population needs and also aids the practicing doctors and hospital service and support staff with timely service with precision there is need for application of ICT to healthcare management system,[ Premkumar and Kosalram 2014].

In the existing system of health care management system, a great amount of manual work has to be done, which requires more number of employees who often take advantage of manual work. A hospital employee cannot immediately satisfy the customer/patient in case of past information because it takes a lot of time to search the information regarding to that query, thus increasing response 
time, [Srilakshmi and Jabasheela, 2014].

Nowadays healthcare organizations globally recognize the importance of applying information technologies to improve the quality of their services and reduce costs,[ Negin et al., 2014].Therefore, it is imperative to assess problems and challenges facing healthcare system and suggests solutions to the problems and challenges using information and communication technology (ICT) via the internet, which is the basis of this research work.

\section{Related Work on Healthcare Management System}

In [Negin et al., 2014], they compared the costs of designing, implementing and running hospital information system with the traditional manual paper method and found that it cost excessively more than the traditional method, they recommended that expensive systems are not applicable for smaller hospitals because of their low income rate. Hence in this research work a low cost hospital information system that applicable for small and large hospitals and even clinics because of its affordability is design and implemented.

In [Srilakshmi and Jabasheela, 2014], they reported that in the existing system of health care industry, a great amount of manual work has to be done, which requires more number of employees who often take advantage of manual work and that a hospital employee cannot immediately satisfy the customer/patient in case of past information because it takes a lot of time to search the information regarding to query, thus increasing response time. Hence they proposed a new system of Hospital Management and Information System using SAP ABAP 4, but ABAP Query needs knowledge of Open SQL commands, this has to be done only by someone who has a little bit of ABAP experience. In a complex reports where data selection are bit complex it may not solve one purpose. A prefer programming language that increase productivity, and overcome the limitation found in the ABAP Programming the .NET environment which offers a unified development environment and a standard set of tools common to all languages and developer tasks is employ in this research work.

In [Nawzat and Yasin, 2014], established that Healthcare Information Systems in hospitals have become an influential factor to provide cooperation among healthcare workers in sharing healthcare information. The research work showed that currently, there is still lack of cooperation among health workers in sharing information and skills in the patients' treatment within the hospital environment. Hence in this research work a low cost hospital information system that allowed cooperation among health workers in sharing information and skills in the patients' treatment within the hospital environment is design and implemented.

In [Jayawardena,2014], found that changing minds of the staff from traditional to new computerization system was the biggest challenge due to most of the staff members thought it is easy to use paper records than computers. In this research work, a user friendly health information system that take into consideration the busy practice of Health workers is design and implemented.

In [Malinga et al., 2014 ] reported that patient dying each year from preventable medical errors, one-fifth of these errors are linked to the lack of prompt access to patient health information. Therefore, it is important to review the literature to determine the best approach to patient health information management and to recommend a model that would address the problems mentioned above. Research and development projects have shown the need for strengthening hospital management information systems (HMIS), patient management information system inclusive, but this has proven to be a difficult task, especially in developing countries [Nawzat and Yasin, 2014]. Orlando's nursing process theory by Orlando (1972) is a suitable guideline for designing a user friendly patient management information system, [Malinga et al., 2014]. Hence in this research work Orlando's deliberative nursing process theory (1972) is used as a suitable guideline for designing a user friendly low cost Healthcare management information system.

\section{Design Methodology}

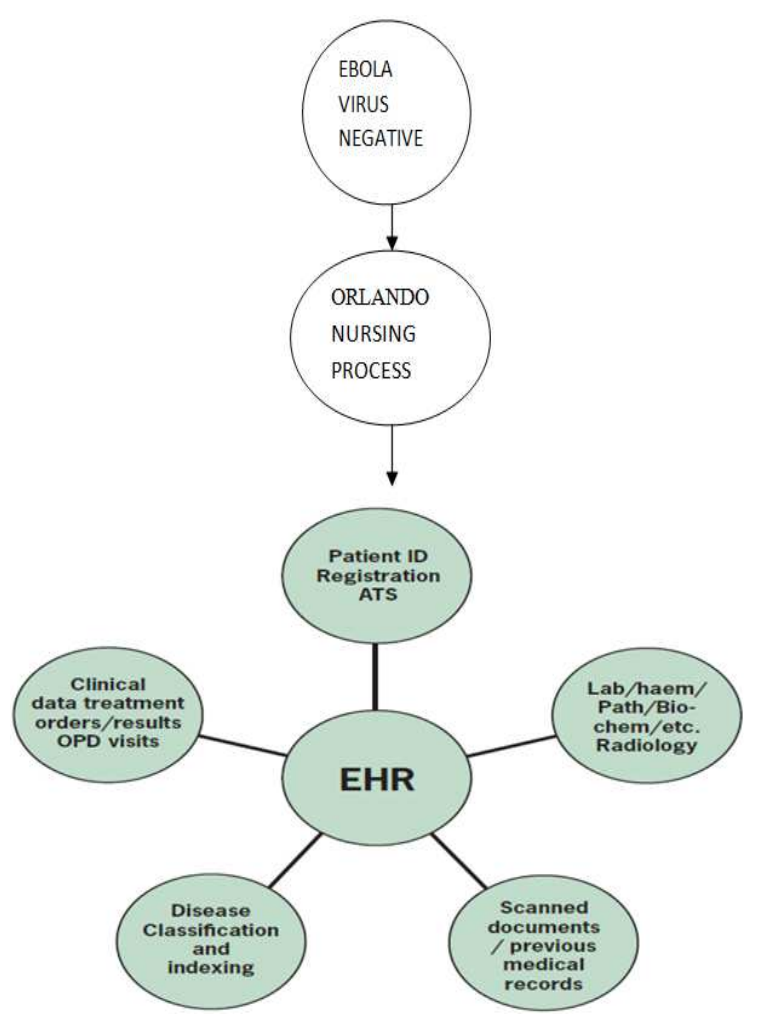

Figure 1. Framework for Orlando nursing process-Ebola virus based model healthcare information management system

This study employed Orlando's nursing process theory (1972) as a suitable guideline for designing a user friendly low cost healthcare management information system which is applicable in any healthcare environment irrespective of their social economic and technology settings. The incorporation Ebola check-up into the framework prevent 
spread of the deadly diseases and it is part of nursing process. It enhances nursing efficiency by standardizing nursing practice and increases care quality through the use of deliberate actions. Healthcare administration requires a large volume of wide-ranging information, and healthcare administrators are limited in their ability to compile and analyze information for healthcare administration. This framework creates methodology for developing a healthcare administration analysis system to aid healthcare administrators in performing outcome analysis, according to the individual needs.

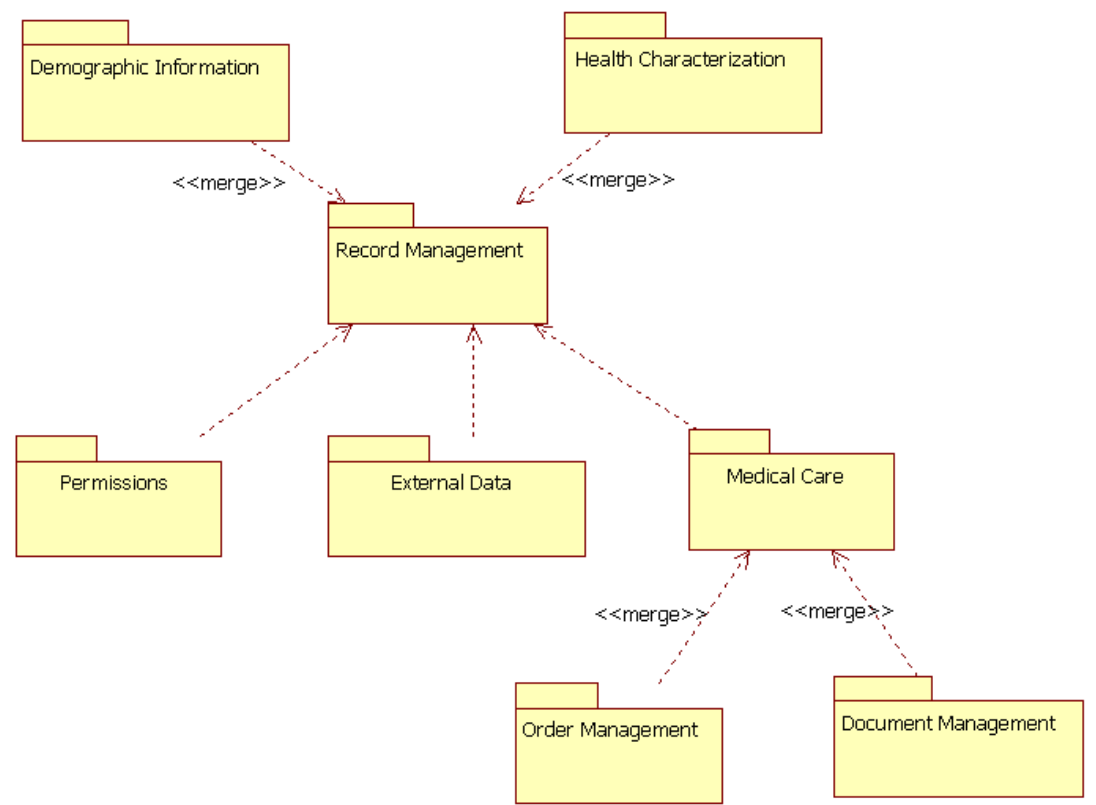

Figure 2. Packages of Patient Information Management System

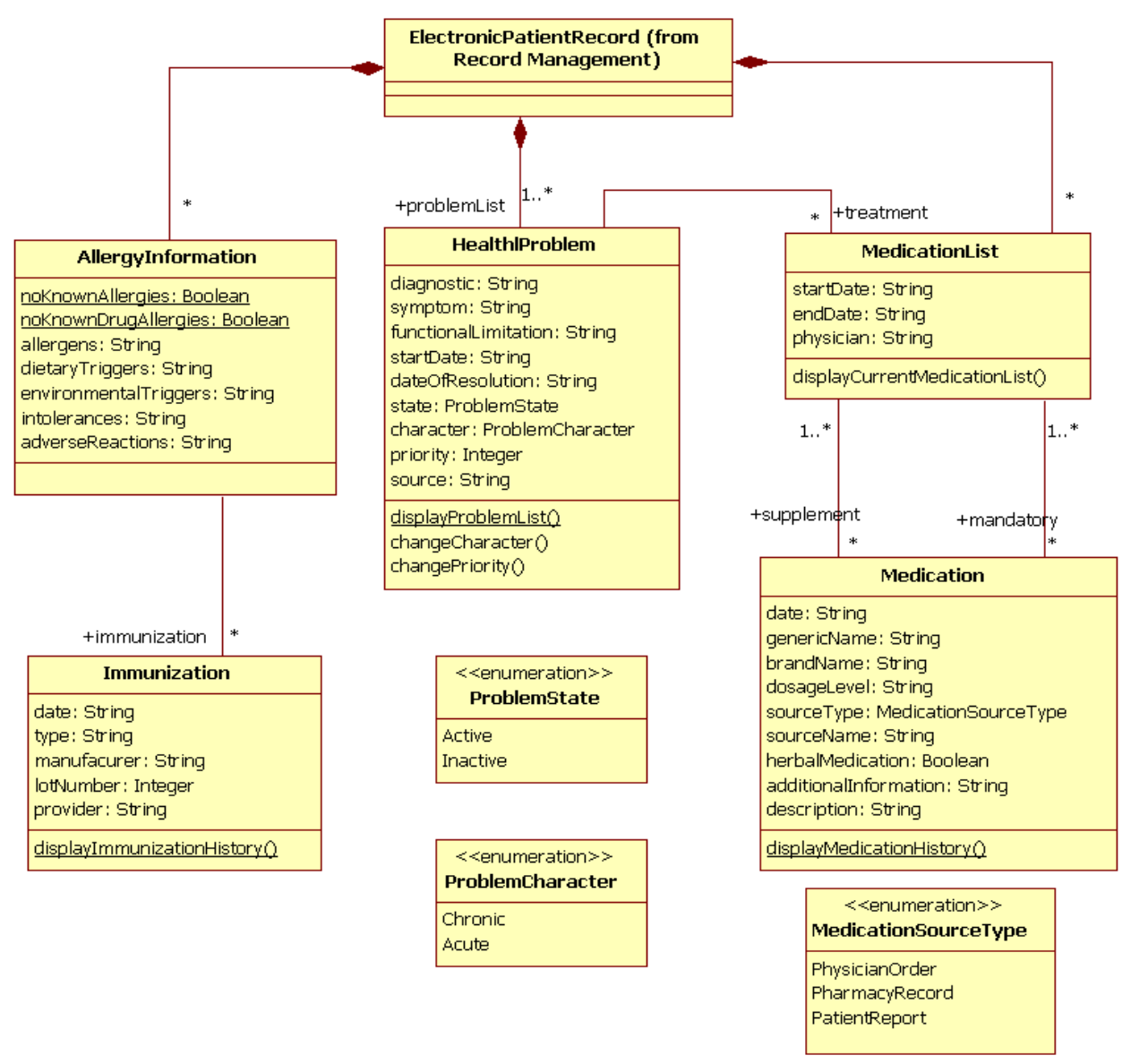

Figure 3. Medical Characterization Package 


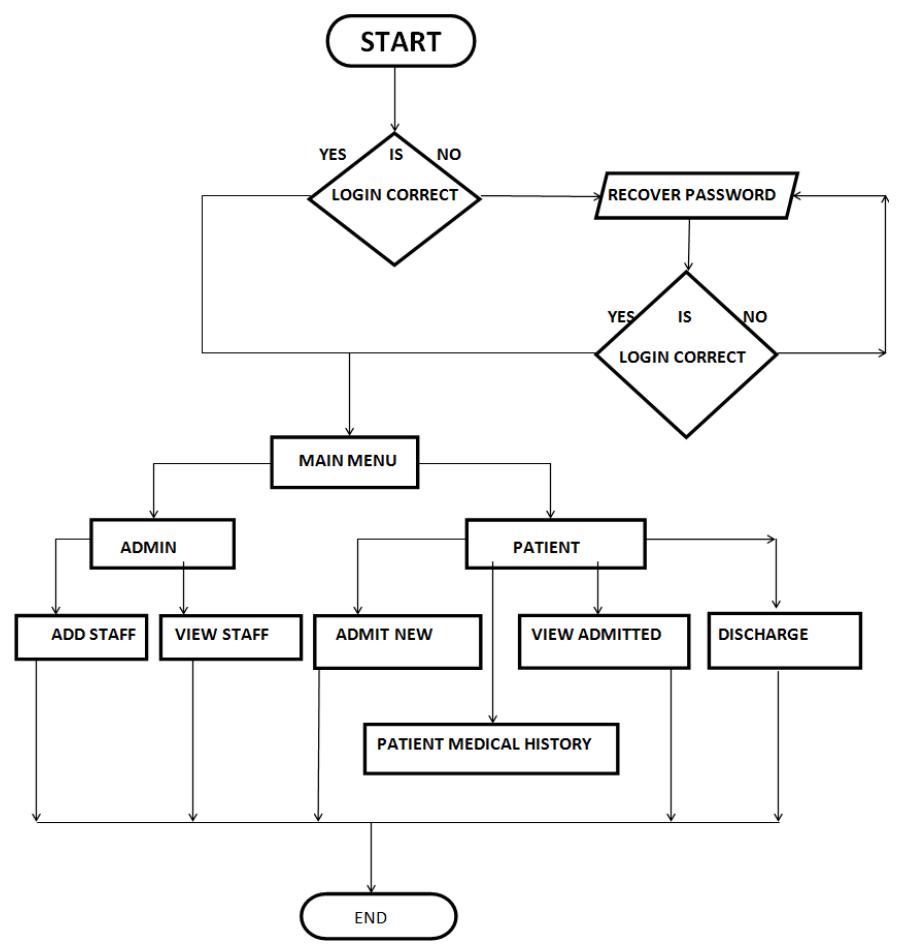

Fig 4. Flowchart of the healthcare Information Management System

\section{Implementation and Results}

VB.net is used as a programming language for the implementation of the design. The programming environment for the database is Microsoft SQL Server 2000 which is connected to VB.net. At the time of admitting the patient, the hospital authorities enter the detailed information of all patients. It includes his/her name, age, sex, and address with phone number etc. It also adds its department, in which the patient is admitted, its bed code, doctor's name, date of admission and the advances taken by hospital. One can get all the information by putting the assigned patient code in the main mouse operated menu bar. During the operational use, the following outputs were obtained according to design modules of Healthcare Management System.

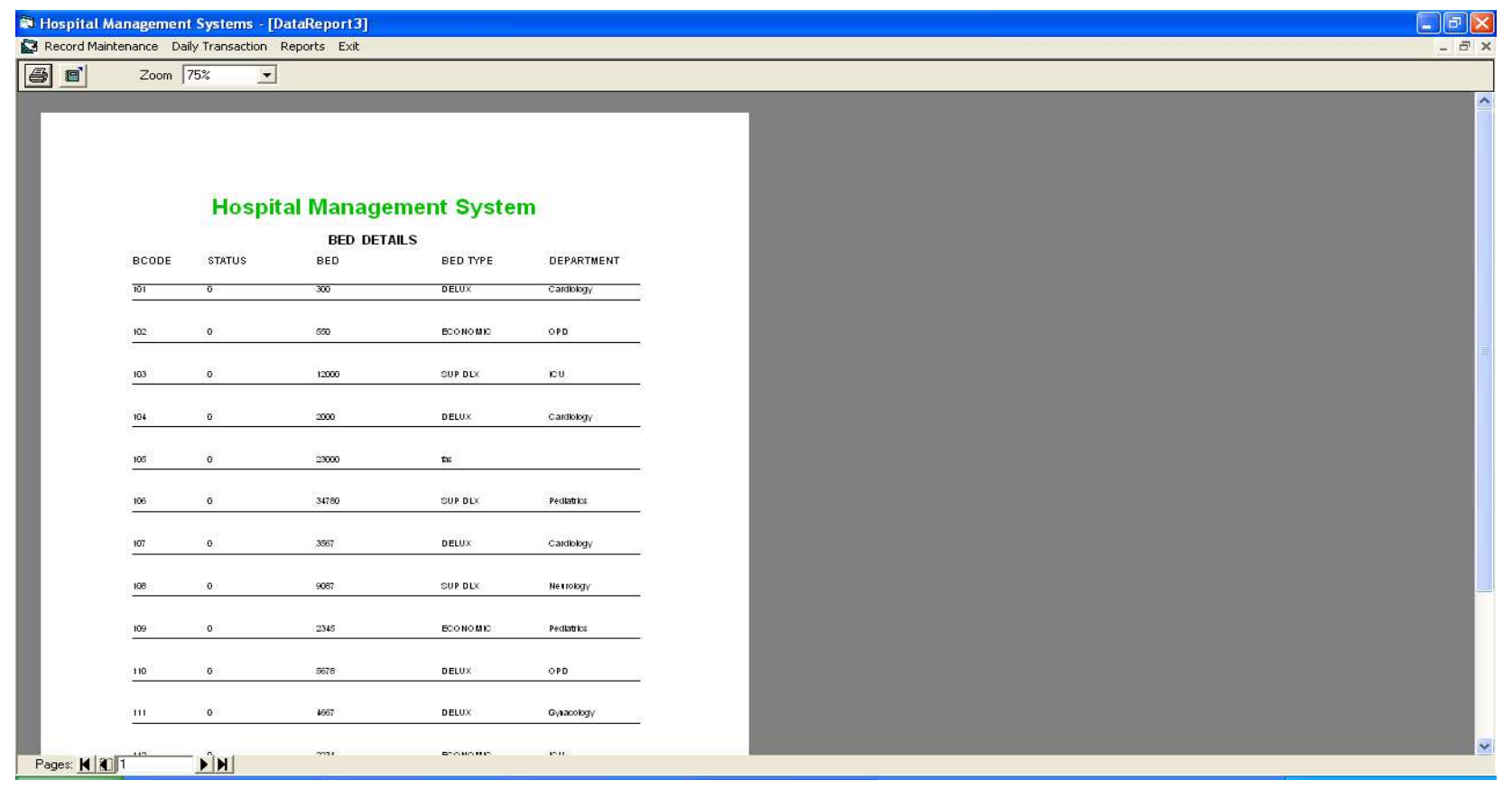

Fig 5. Patient Details 


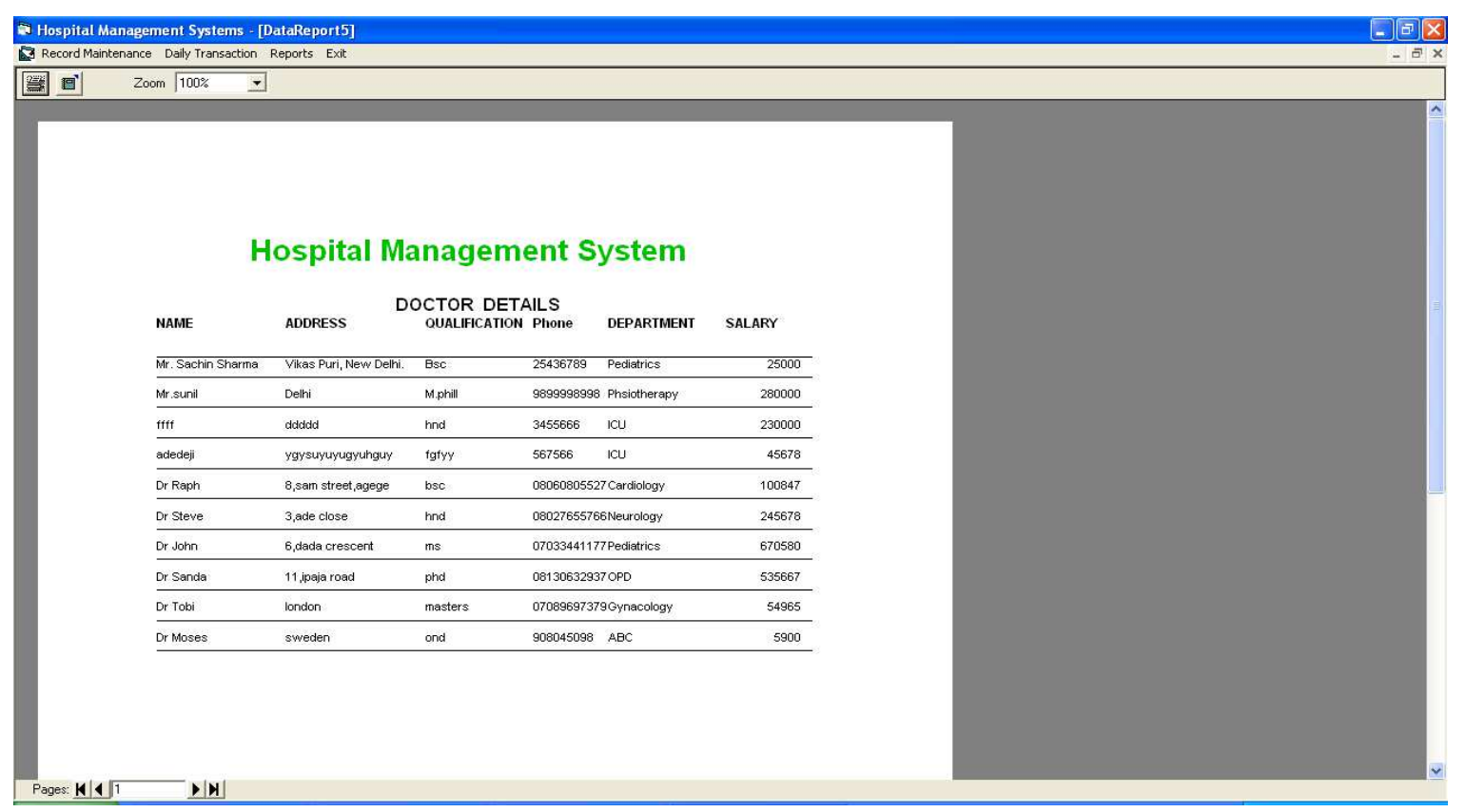

Fig 6. Doctors Details

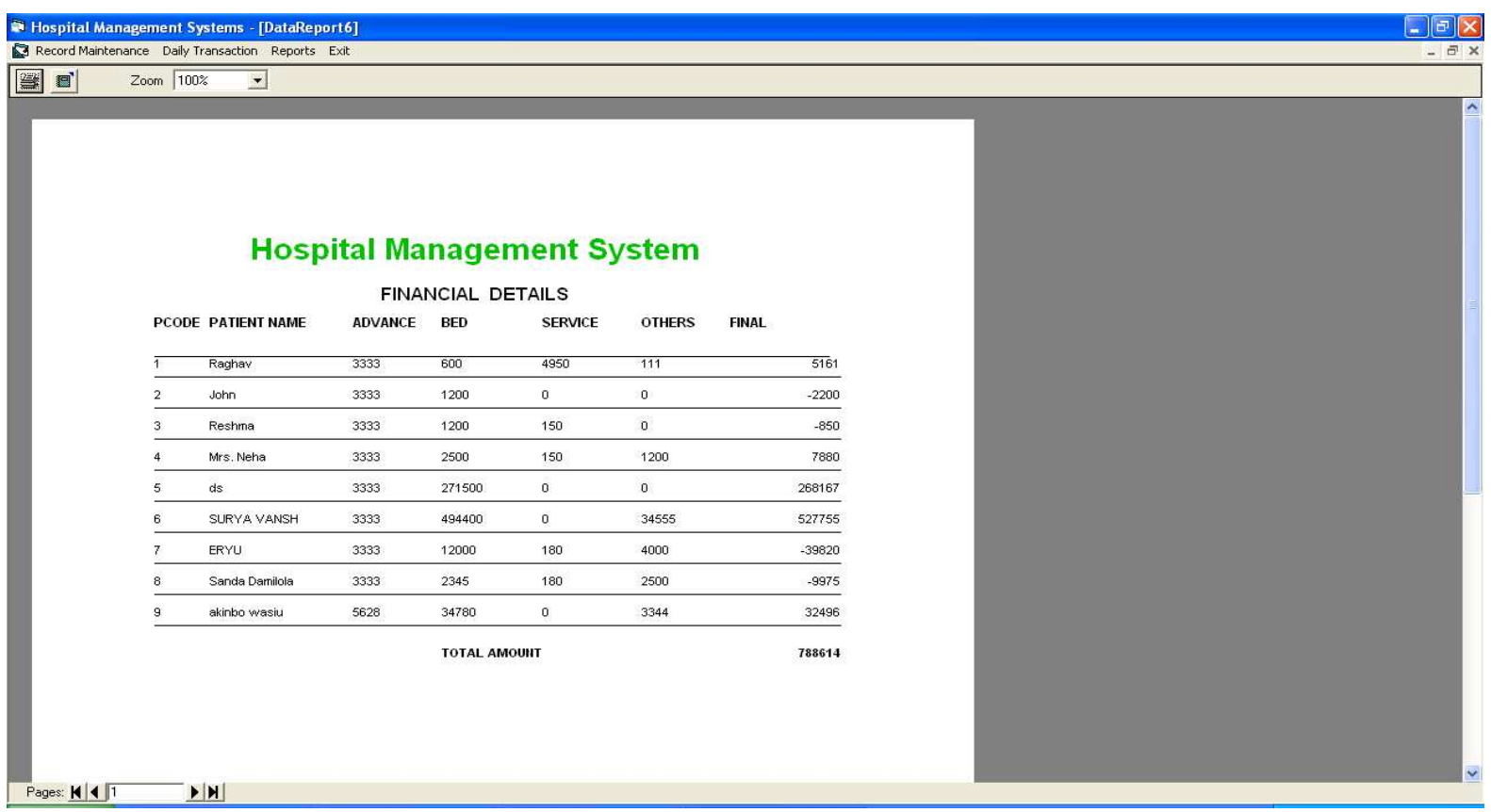

Fig 7. Financial Details

\section{Evaluation of the Healthcare Management System}

\subsection{Research Design}

The descriptive survey design was adopted which involved the collection of primary and cross sectional data through the use of a structured questionnaire. A preliminary study visit was made to Rabiat Shoaga Triumphant Health Centre Abeokuta Nigeria in April 2014 to find out about the feasibility of the study. The sample frame for this study comprised exhaustive list of the Healthcare Centre units. The purposive sampling method was adopted in selecting the respondents so as to ensure that selected individuals were those that had adequate knowledge of healthcare services.

\subsection{Instrument for Data Collection}

Data were collected with a structured questionnaire designed in a four point Like scale, comprised four sections: Section A elicited information about units in the Healthcare Centre. Section B asked questions about Health resources available in each Unit. While section $\mathrm{C}$ sought to ascertain the stage of Healthcare Global Standard in the Health Centre. Section D, the last section, contained questions that enquired 
about the challenges encountered by the units in Healthcare information System adoption and implementation processes. The instrument was validated through face and content validity. It was subjected to thorough scrutiny by three experts in Healthcare information System research and two others in the field of information science. Modifications were made on the instrument based on their assessments. Copies of the questionnaire were distributed to the respondents by the researchers who had initially sought the permission of the Director of the Healthcare Centre. A total of 145 copies of the questionnaire were distributed but 135 copies were completed and returned. This constituted $93.10 \%$ and was used for data analyses.

\subsection{Data Analysis}

The Statistical Package for Social Science (SPSS) software was used to carry out the analysis. The variables used to assess the Healthcare Information system using 4-point like scale were re-coded. Strongly Agree and Agree were recoded as High while Disagree and strongly Disagree were recoded as Low. Next, a frequency distribution tables was generated for all the variables.

Table 1. Distribution of variables concerns the Healthcare Information System

\begin{tabular}{|c|c|c|c|c|c|c|c|c|c|c|c|}
\hline & & \multicolumn{2}{|c|}{ Ease of Use } & \multicolumn{2}{|c|}{ Sustainability } & \multicolumn{2}{|c|}{ Operational Efficiency } & \multicolumn{2}{|c|}{ Data Security } & \multicolumn{2}{|c|}{ Human factors } \\
\hline & & Freq. & $\%$ & Freq & $\%$ & Freq & $\%$ & Freq & $\%$ & Freq. & $\%$ \\
\hline \multirow[t]{3}{*}{ Valid } & Low & 25 & 18.51 & 28 & 20.74 & 21 & 15.56 & 14 & 10.37 & 23 & 17.04 \\
\hline & High & 110 & 81.49 & 107 & 79.26 & 114 & 84.44 & 121 & 89.63 & 112 & 82.96 \\
\hline & Total & 135 & 100 & 135 & 100 & 135 & 100 & 135 & 100 & 135 & 100 \\
\hline
\end{tabular}

The distribution of variables as relates to the healthcare information system is as presented in table 1.The number of user that interacted with the system and reported that the system is easy to use, its operation is efficient and data are protected and secured are more than $80 \%$. While sustainable of the system is $79.26 \%$, this may due to the fact that user perceive, if required infrastructure is not maintain and upgrade in future this may affect the system. Human or Individual factor is $82.96 \%$ this is due to ease of use that make user to easily acquired sufficient knowledge and skills on the use of the system and result to low resistance to change from traditional ways of doing things. A total of 145 copies of the questionnaire were distributed but 135 copies were completed and returned. The system shows 135/145 $(93.10 \%)$ participation. From the evaluation, we can conclude that (HIS) is highly efficient, effective and satisfactory to the target users (health stakeholders).

\section{Discussion and Recommendation}

The processes and management of healthcare records are not trivial exercise, healthcare workers practitioners' needs accurate and timely information concerns patients in order to save life, and performed their duties professionally. The framework and healthcare information system presents in this research works will offer a standard approach in developing cooperation among healthcare workers with regard to sharing healthcare information and performed their duties professionally through the application of ICT and Orlando nursing process in the healthcare services. The study offers a demonstration of the application of ICT tools and Orlando's nursing process nursing process to improved healthcare services. The framework and Healthcare Management System are adequate enough to address global demands in health service delivery irrespective of socialeconomic and technology set-up of the healthcare setup making use of them. It is user friendly and low cost healthcare management information system.
Today's Healthcare Information are in high demand in order to handle increasing population needs and also aids the practicing health workers, health services and support staff with timely service with precision, in design and implementation of this Healthcare Management System the busy practice of Health workers is put into consideration.

The Healthcare Management System was evaluated with usability testing, by allowing users to run through the system with real life data and structured questionnaire. The results show $(9.9 \%)$. The number of user that interacted with the system and reported that the system is easy to use, its operation is efficient, stored data are protected and secured are more than $80 \%$. While sustainable of the system is $79.26 \%$, this may due to the fact that user perceive, if required infrastructure is not maintain and upgrade in future this may affect the system. Human or Individual factor is $82.96 \%$ this is due to ease of use the Healthcare Information System that make user to easily acquired sufficient knowledge and skills on the use of the system and result to low resistance to change from traditional ways of doing things. From the evaluation, we can conclude that (HIS) is highly efficient, effective and satisfactory to the target users (health stakeholders). The application of the framework will prevents the spread of deadly diseases like Ebola Virus.

This system is recommended to any healthcare service provider that wishes to move its service forward by healthcare global standard. The basic infrastructure that will make such a system work should be provided and made affordable by the healthcare provider.

\section{References}

[1] Jayawardena AS (2014) The Electronic Hospital Information System Implemented at the District General Hospital Trincomalee-An Experience of Business Process Reengineering. J Community Med Health Educ S2: 001. doi:10.4172/2161-0711.S2-001 
[2] Igira, F., T., Titlestad, O., H., Lungo, J., H., Makungu, A., Khamis, M., M., Sheikh, Y., Mahundi, M., Ngeni, M., J., Suleiman., \& Braa, J.(2009). Designing and Implementing Hospital Management Information Systems in Developing Countries: Case Studies from Tanzania - Zanzibar http://www.sim.hcuge.ch/helina/19.pdfPp 77-83

[3] Malinga Ramadhan B.*, Ssenyonga T., and Novembrieta R. Sumil (2014) Development and implementation of patient management information system of kampala international university teaching hospital(kiu-th), bushenyi district, Uganda Journal of Poverty, Investment and Development - An Open Access International Journal Vol.4 2014,Pp. 77-83.

[4] Nawzat Sadiq Ahmed and Norizan Mohd Yasin (2014): Factors affecting cooperation among physicians in sharing information within the hospital environment: a study of two hospitals. Journal of Computer Science 10 (5): 794-808, 2014.Pp794-808.

[5] Negin Karimi Hosseini, Md Jan Nordin, Mitra Mahdiani and Samira Sadrzadeh Rafiei (2014): A Low Cost Hospital
Information System: A Case Study of the Mehr Hospital in Mashhad City. Research Journal of Applied Sciences, Engineering and Technology 2014.Pp1132-1138.

[6] Premkumar Balaraman and Kalpana Kosalram (2014): E Hospital Management and Hospital Information Systems Changing Trends. International journal of Information Engineering and Electronic Business, 2013, 1Pp. 50-58.

[7] SrilakshmiSatyapraba J and L.Jabasheela (2014): Hospital Management and Information System Using SAP,International journal of advanced computer technology a Special Issue of 4th National Conference on Advanced Computing, Applications \& Technologies, May 2014.Pp21-26.

[8] Yang, H., K. Liu and W. Li, 2010.Adaptive requirementdriven architecture for integrated healthcare systems. J. Comput., 5:Pp. 186-193.

[9] Yang, T.H., Y.S. Sun and F. Lai, 2011. A scalable healthcare information system based on a service oriented architecture. J. Med. Syst., 35: 391-407. 